\title{
Correction to: CCZ equivalence of power functions
}

\author{
Ulrich Dempwolff ${ }^{1}$
}

Accepted: 10 November 2021 / Published online: 12 December 2021

(c) Springer Science+Business Media, LLC, part of Springer Nature 2021

\section{Correction to: Designs, Codes and Cryptography (2018) 86:665-692 https://doi.org/10.1007/s10623-017-0350-8}

\begin{abstract}
An error in the proof of Lemma 2.13 of my article [3] is corrected. We point out that the validity of the main result [3, Theorem 1.1$]$ is not affected.
\end{abstract}

The correct form of [3, Lemma 2.13] is:

Lemma 1 Let $0<k<p^{n}-1$, $k$ not a p-power. Then:

(a) $C_{\text {Aut }\left(p_{k}\right)}\left(\mathcal{Z}_{k}\right)=\mathcal{Z}_{k}$.

(b) Either $\mathcal{N}_{k}$ is the common stabilizer of the spaces $V$ and $W$ in $\operatorname{Lin}\left(p_{k}\right)$ or $n=4 b$, $k \equiv p^{a}\left(1+p^{b}\right)\left(\bmod p^{n}-1\right)$ for some integers $a, b$ and cyclic groups of order $p^{n}-1$ in $\operatorname{Aut}\left(p_{k}\right)$ are conjugate to $\mathcal{Z}_{k}$.

The author missed the second part of assertion (b) of Lemma 1. The second part of assertion (b) does not change the validity of the main result [3, Theorem 1.1]: To see this consider power functions $p_{k}$ and $p_{\ell}$ on $\mathrm{GF}\left(p^{n}\right)$ which are CCZ equivalent. One has to show that there exists an integer $a$ such that $\ell \equiv p^{a} k\left(\bmod p^{n}-1\right)$ or $k \ell \equiv p^{a}\left(\bmod p^{n}-1\right)$ (in which case $k$ is invertible modulo $\left.p^{n}-1\right)$. If $n=4 b$ and $k \equiv p^{a}\left(1+p^{b}\right)\left(\bmod p^{n}-1\right)$ then by the second part of assertion (b) of Lemma 1 and [3, Lemma 2.23] we get $\ell \equiv p^{c} k\left(\bmod p^{n}-1\right)$ as $k$ is not invertible modulo $p^{n}-1$. In particular the assertion of the main result holds. So one can assume from now on $k, \ell \not \equiv p^{a}\left(1+p^{b}\right)\left(\bmod p^{n}-1\right)$ and $\mathcal{N}_{k}$ is the common stabilizer of the spaces $V$ and $W$ in $\operatorname{Lin}\left(p_{k}\right)$; i.e. [3, Lemma 2.13] can be applied in it's original form and we can complete the proof of [3, Theorem 1.1] following the arguments of [3].

We recall the notation from [3]. Set $F=\mathrm{GF}\left(p^{n}\right), p$ a prime, $n$ a positive integer. Let $k$ be a non-negative integer $<p^{n}-1$. Then $p_{k}: F \rightarrow F^{\prime}$ denotes the power function on $F$ defined by $p_{k}(x)=x^{k}$ and $F^{\prime}$ stands for the subfield of $F$ generated by the image of $p_{k}$. Set $U=V \oplus W$,

Communicated by A. Pott.

The original article can be found online at https://doi.org/10.1007/s10623-017-0350-8.

Ulrich Dempwolff

dempwolff@mathematik.uni-kl.de

1 Department of Mathematics, University of Kaiserslautern, Erwin-Schroedinger-Strasse, 67653

Kaiserslautern, Germany 
$V=F \oplus 0, W=0 \oplus F^{\prime}$. Then $U$ contains the graph $\Gamma\left(p_{k}\right)=\left\{\left(x, p_{k}(x)\right) \mid x \in F\right\}$ of $p_{k}$. The automorphism group of $p_{k}$ (in the sense of CCZ equivalence) is the stabilizer of $\Gamma\left(p_{k}\right)$ in $\operatorname{AGL}(U): \operatorname{Aut}\left(p_{k}\right)=\left\{\Phi \in \operatorname{AGL}(U) \mid \Gamma\left(p_{k}\right) \Phi=\Gamma\left(p_{k}\right)\right\}$. Here $\operatorname{AGL}(U)$ denotes the group of invertible affine transformations on $U$, i.e. a typical element of $\operatorname{AGL}(U)$ has the form $\phi \circ \tau_{u}$ with $\phi \in \mathrm{GL}(U)$ and $\tau_{u}$ is the translation $\tau_{u}: U \ni x \mapsto x+u \in U$. The group $\operatorname{Lin}\left(p_{k}\right)=\operatorname{Aut}\left(p_{k}\right) \cap \operatorname{GL}(U)$ is the stabilizer of 0 in $\operatorname{Aut}\left(p_{k}\right)$. For $0 \neq b \in F$ define $\mu_{b} \in \mathrm{GL}(U)$ by $(x, y) \mu_{b}=\left(b x, b^{k} y\right)$. Define $\phi \in \operatorname{GL}(U)$ by $(x, y) \phi=\left(x^{p}, y^{p}\right)$. Then the groups $\mathcal{Z}_{k}=\left\{\mu_{b} \mid 0 \neq b \in F\right\}$ and $\mathcal{N}_{k}=\mathcal{Z}_{k}\langle\phi\rangle$ are subgroups of $\operatorname{Lin}\left(p_{k}\right)$.

Keeping the notation of [3] we revise the proof of part (b) and use the following lemma. Note, that we consider in this lemma isomorphisms of $\mathrm{GF}(p) G$-modules although these modules may be defined as G-modules over larger fields.

Lemma 2 Let $G=\operatorname{SL}\left(d, p^{a}\right), d \geq 2,\left(d, p^{a}\right) \neq(2,2),(2,3)$ and denote by $M$ the natural $F G$-module, $F=\mathrm{GF}\left(p^{a}\right)$. Set $n=$ ad and let $N$ be a simple $\mathrm{GF}(p) G$-module of dimension $n$. One of the following $\mathrm{GF}(p) G$-isomorphisms holds:

(i) $N$ is isomorphic to $M$,

(ii) $d>2$ and $N \simeq M^{*}$ (the dual module of $M$ ),

(iii) $d=2, a=2 b$, and $N$ is isomorphic to a $\mathrm{GF}\left(p^{b}\right) G$-submodule $R$ of $M \otimes M^{\left(p^{b}\right)}$, $\operatorname{dim}_{\mathrm{GF}\left(p^{b}\right)} R=4$.

We denote by $M^{\left(p^{c}\right)}$ the Galois conjugate of a module $M$ under the field automorphism $x \mapsto x^{p^{c}}$.

Proof Set $F_{0}=\operatorname{End}_{\mathrm{GF}(p) G} N$. Then $F_{0} \simeq \mathrm{GF}\left(p^{b}\right)$ is a finite field (Schur's Lemma). By [1, (25.8)] $N$ is an absolutely irreducible $F_{0} G$-module. By [5, Theorem 2.1] $\operatorname{dim}_{F_{0}} N \geq d^{a /(a, b)}$. So $a d=n=\operatorname{dim}_{\mathrm{GF}(p)} N \geq b d^{a /(a, b)}$. Two possibilities can occur: $a=b$ or $d=2, a=2 b$. Set $N^{F}=F \otimes_{F_{0}} N$ which is simple as a $F G$-module. If $a=b$ then $\operatorname{dim}_{F} N^{F}=d$ and by [6, Theorem 2.2] assertion (i) or (ii) holds as Galois conjugates of $F G$-modules are isomorphic as $\operatorname{GF}(p) G$-modules. Case $a=2 b$ yields assertion (iii): Now $d=2$ and $\operatorname{dim}_{F} N^{F}=4$ so that [6, Theorem 2.2] implies $N^{F} \simeq M \otimes M^{\left(p^{c}\right)}$ (as $\operatorname{GF}(p) G$-modules) for some $c$. However $N^{F}$ as a $F_{0} G$-module is the direct sum of two isomorphic, simple modules (which we call $R)$ and $\left(N^{F}\right)^{\left(p^{b}\right)} \simeq N^{F}$. This forces $b=c$.

Proof of $(b)$ Let $L$ be the stabilizer of the spaces $V$ and $W$ in $\operatorname{Lin}\left(p_{k}\right)$. Let $\pi: L \rightarrow \operatorname{GL}(V)$ be the projection of $L$ into GL(V). Then $\operatorname{ker} \pi=1$ by [3, Lemma 2.4]. By [4] $L \simeq \operatorname{Im} \pi \simeq$ $\Gamma \mathrm{L}\left(d, p^{a}\right), a d=n$, and $V$ is the natural $F L$-module, $F=\mathrm{GF}\left(p^{a}\right)$, viewed as a module over $\operatorname{GF}(p)$. The group $L$ contains the normal subgroups $\operatorname{SL}\left(d, p^{a}\right) \simeq L_{0} \leq L_{1} \simeq \operatorname{GL}\left(d, p^{a}\right)$. The first assertion of (b) holds if $d=1$.

So assume $d>1$. If the projection of $L_{0}$ into $\operatorname{GL}(W)$ is trivial, then $p_{k}$ is constant $\left(L_{0}\right.$ is transitive on $V-\{0\})$ and $k=0$, a contradiction.

So let the the projection of $L_{0}$ into $\operatorname{GL}(W)$ be nontrivial. We consider $V$ and $W$ as $\mathrm{GF}(p) L_{0}$-modules and distinguish the three cases of Lemma 2.

Assume, that $V \simeq W$ as $\mathrm{GF}(p) L_{0}$-modules. By [3, Lemma 2.3] the nontrivial $L_{0}$-orbits consist of the nontrivial elements of an $n$-space and $k$ would be a $p$-power by [3, Lemma 2.11], a contradiction. Assume next, that $d>2$ and that the modules $V$ and $W$ are dual to each other. Then the stabilizer of a vector on $V$ does not fix any nontrivial vector in $W$, i.e. this group does not have the orbit $B=\Gamma\left(p_{k}\right)-\{0\}$ of length $p^{n}-1$ in $U-(V \cup W)$, a contradiction.

Finally, we assume (notation as in Lemma 2) $d=a / b=2, V \simeq M$, and $W \simeq R$ as $\mathrm{GF}(p) L_{0}$-modules. This case was missed in the proof of [3, Lemma 2.13]. Set $F_{0}=\operatorname{GF}\left(p^{b}\right)$. 
We know that $V$ is the natural module of $L_{1}$ and in the the obvious way one extend the $F_{0} L_{0}$ module $W \simeq M \otimes M^{\left(p^{b}\right)}$ and even $R$ to a $F_{0} L_{1}$-module. Note, that if $L_{1}$ acts in this form on $W$ there exist orbits of length $p^{n}-1$ in $U-(V \cup W)$ (the stabilizer in $L_{1}$ of a nontrivial vector of $V$ fixes in $W$ a nontrivial vector too).

Any $F_{0} L_{1}$-module whose restriction to $L_{0}$ is isomorphic to $M \otimes M^{\left(p^{b}\right)}$ is of the form $\left(M \otimes M^{\left(p^{b}\right)}\right) \otimes \chi$ where $\chi: L_{1} / L_{0} \rightarrow F_{0}^{*}$ is a linear character. If $W \simeq R$ as a $\operatorname{GF}(p) L_{1}$ is a subrepresentation of $\left(M \otimes M^{\left(p^{b}\right)}\right) \otimes \chi$ then $U-(V \cup W)$ contains $L_{1}$-orbits of length $p^{n}-1$ only if $\chi=1$. This shows $W \subset M \otimes M^{\left(p^{b}\right)}$ as a $\operatorname{GF}(p) L_{1}$ module. Let $\zeta$ be a primitive element in $F$. Then $\mu_{\zeta}$ has on $F \otimes V$ the eigenvalues $\zeta, \zeta^{p}, \ldots, \zeta^{p^{n-1}}$, on $F \otimes W$ the eigenvalues $\zeta^{k}, \zeta^{p k}, \ldots, \zeta^{p^{n-1} k}$ and on $M \otimes M^{\left(p^{b}\right)}$ the eigenvalues $\zeta^{1+p^{\zeta}}, \zeta^{p\left(1+p^{b}\right)}, \ldots, \zeta^{p^{n-1}\left(1+p^{b}\right)}$. Hence $k=p^{a}\left(1+p^{b}\right)$ for some $a$, i.e. $p_{k}$ is a quadratic function. Set $v(c)=\beta(c) \circ \tau_{\left(c, p_{k}(c)\right)} \in \operatorname{AGL}(U), c \in F$, where $\beta(c) \in \operatorname{GL}(U)$ is defined by $(x, y) \beta(c)=\left(x, p_{k}(x+c)+p_{k}(x)+y\right)$. Then $\mathcal{M}=\{v(c) \mid c \in F\}$ is an elementary abelian $p$-group, that acts regularly on $\Gamma\left(p_{k}\right)$. A computation shows that $\mathcal{Z}_{k}$ normalizes $\mathcal{M}$. So $\mathcal{M} \cdot \mathcal{Z}_{k} \leq G=\operatorname{Aut}\left(p_{k}\right)$ acts doubly transitive on $\Gamma\left(p_{k}\right)$. The socle of $G$ is either an elementary abelian subgroup of order $p^{n}$ or is a nonabelian simple group.

Suppose first that the socle $\mathcal{M}_{1}$ of $G$ is elementary abelian. Then $C_{\mathcal{M}_{1}}(\mathcal{M}) \neq 1$ is invariant under $\mathcal{Z}_{k}$ showing $C_{\mathcal{M}_{1}}(\mathcal{M})=\mathcal{M}_{1}$ and therefore $\mathcal{M}=\mathcal{M}_{1}$. Now $G=\mathcal{M} \cdot \operatorname{Lin}\left(p_{k}\right)$ and as $L \leq \operatorname{Lin}\left(p_{k}\right)$ we deduce from a Theorem of Hering (see [6, Appendix]) GL(e, $\left.p^{c}\right) \leq$ $\operatorname{Lin}\left(p_{k}\right) \leq \Gamma \mathrm{L}\left(e, p^{c}\right), e \geq d, e c=n$. The well known Theorem of Zsigmondy implies that cyclic groups of order $p^{n}-1$ of $\operatorname{GL}(n, p)$ are Singer groups and [4] shows that cyclic groups of order $p^{n}-1$ of a subgroup of $\operatorname{GL}(n, p)$ form a conjugacy class in this subgroup. Therefore a cyclic subgroup of $G / \mathcal{M}$ of this order is conjugate to $\mathcal{Z}_{k} \mathcal{M} / \mathcal{M}$ and the Schur-Zassenhaus Theorem in turn implies the second assertion of $(b)$.

Suppose now that the socle $S$ of $G$ is a nonabelian simple group. Then $S$ is listed in [2, Theorem 5.3]. Note, that $S$ has prime power degree and a point stabilizer contains a group isomorphic to $\operatorname{SL}\left(2, p^{a}\right)$. A quick inspection of the examples shows $S \simeq \operatorname{Alt}\left(p^{n}\right)$. As $p^{n} \geq 16$ the degree of a minimal nontrivial projective representation of $\operatorname{Alt}\left(p^{n}\right)$ is $\geq p^{n}-2$ (see [7,8]). But the image of $S$ under the epimorphism $\operatorname{AGL}(U) \rightarrow \operatorname{GL}(U)$ has a nontrivial representation of degree $2 n$, a contradiction.

\section{References}

1. Aschbacher M.: Finite Group Theory. Cambridge University Press, Cambridge (2000).

2. Cameron P.: Primitive permutation groups and finite simple groups. Bull. Lond. Math. Soc. 13, 1-22 (1981).

3. Dempwolff U.: CCZ equivalence of power functions. Des. Codes Cryptogr. 86, 665-692 (2018).

4. Kantor W.: Linear groups containing a Singer cycle. J. Algebra 62, 232-234 (1980).

5. Liebeck M.: On the orders of maximal subgroups of the finite classical groups. Proc. Lond. Math. Soc. (3) 50, 426-446 (1985).

6. Liebeck M.: The affine permutation groups of rank three. Proc. Lond. Math. Soc. (3) 54, 477-516 (1987).

7. Wagner A.: The faithful linear representation of least degree of $S_{n}$ and $A_{n}$ over a field of characteristic 2 . Math. Z. 151, 127-137 (1976).

8. Wagner A.: An observation on the degrees of projective representations of the symmetric and alternating group over an arbitrary field. Arch. Math. (Basel) 29, 583-589 (1977).

Publisher's Note Springer Nature remains neutral with regard to jurisdictional claims in published maps and institutional affiliations. 